\title{
NKX2.1 (TTF1) germline mutation associated with pulmonary fibrosis and lung cancer
}

\begin{abstract}
To the Editor:
Copyright (CThe authors 2021

This version is distributed under the terms of the Creative Commons Attribution Non-Commercial Licence 4.0. For commercial reproduction rights and permissions contact permissions@ersnet.org

Received: 27 May 2021 Accepted: 27 July 2021

\section{○ $\odot \oplus$}

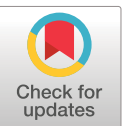

The high prevalence of lung cancer in patients with idiopathic pulmonary fibrosis (3-30\%) has been confirmed by several studies, pointing to specific diagnostic and therapeutic issues. The co-occurrence is associated with worse survival than with each disease alone [1]. Because cigarette smoking is a risk factor for both diseases, smoking is an ideal culprit for their co-occurrence, despite several common pathogenic mechanisms such as common genetic risk factors.

Several germline mutations have been associated with pulmonary fibrosis [2]. Mutation of telomere-related genes is the most frequent (25-30\% of familial cases) [2], with mutation of surfactant-associated genes (including SFTPC (location: 8p21.3), SFTPB (2p11.2), SFTPA1 (10q22.3), SFTPA2 (10q22.3), ABCA3 (16p13.3) and NKX2.1 (14q13.3)) second in frequency (1-5\% of familial cases) [2]. SFTPA1 and SFTPA2 germline mutations are unique in that they are associated with a high prevalence of lung cancer, although the mechanisms are poorly understood [3].

The NKX2.1 gene codes for thyroid transcription factor 1 (TTF1), which is implicated in lung development and the expression of surfactant proteins [4]. NKX2.1 heterozygous mutations have been associated with "brain-lung-thyroid syndrome", characterised by central nervous system abnormalities, hypothyroidism and interstitial lung disease (ILD), with an inconstant triad [5]. Severe ILD can be the only manifestation in up to $25 \%$ of $N K X 2.1$ mutation carriers [5]. Only three patients with lung cancer associated with NKX2.1 mutation have been reported, with few data (figure 1a) [6]. Here, we describe a woman with lung fibrosis and chorea associated with NKX2.1 mutation, complicated by lung cancer.

A 42-year-old woman, without previous respiratory symptoms, was referred to our department in 2014 for a diagnosis of ILD. She had a history of chorea and subclinical hypothyroidism. The chorea led to the identification, at age 32 years, of a de novo c.267dupG NKX2.1 mutation [9]. Neither of her parents had ILD, chorea or hypothyroidism, and neither carried the mutation. The patient received prenatal screening during pregnancy and none of her asymptomatic children carried the mutation. She had a 10-pack-year smoking history and no other toxic lung exposures. She did not present any clinical or biological signs of an autoimmune disease. Clinical evaluation showed lung crackles and a few abnormal involuntary movements. Laboratory test results were within the normal range. High-resolution chest computed tomography (CT) revealed a pattern indeterminate for usual interstitial pneumonia, with honeycombing, ground-glass opacities, reticulations and traction bronchiectasis with ventral and basal predominance (figure 1b-e). Because the distribution of lung fibrosis did not suggest any specific aetiology, the chest CT was considered truly indeterminate and not suggestive of an alternative diagnosis. Bronchoalveolar lavage analysis revealed 335000 cells per mL (68\% macrophages, 10\% lymphocytes, 15\% neutrophils and 7\% eosinophils) and Golde score 46 (which evaluates haemosiderin-laden macrophages, a score >100 suggesting diffuse alveolar haemorrhage). Bronchial biopsy showed mild, nonspecific bronchial inflammation.

Surgical lung biopsy was declined considering the severity of the disease at diagnosis: forced vital capacity $1.36 \mathrm{~L}$ (46\% predicted) and diffusing capacity of the lung for carbon monoxide $26 \%$ predicted. On right heart catheterisation, mean pulmonary artery pressure was $40 \mathrm{mmHg}$, pulmonary capillary wedge pressure

\section{Shareable abstract (@ERSpublications) cancer https://bit.ly/3CkkXgD}

Germline surfactant-associated genes mutations are associated with ILD and increased risk of lung

Cite this article as: Borie R, Funalot B, Epaud R, et al. NKX2.1 (TTF1) germline mutation associated with pulmonary fibrosis and lung cancer. ERJ Open Res 2021; 7: 00356-2021 [DOI: 10.1183/ 23120541.00356-2021].
\end{abstract}


a)

\begin{tabular}{|c|c|c|c|c|}
\hline & GRAS et al. [6] & WiLLEMSEN et al. [7] & GLIK et al. [8] & This case \\
\hline Chorea & Since childhood & Since childhood & Since childhood & Since childhood \\
\hline Hypothyroidism & Yes (age 40 years) & Yes (age 15 months) & Unknown & Yes (age 36 years) \\
\hline ILD & Unknown & $\begin{array}{l}\text { Pulmonary alveolar proteinosis (age } 11 \text { months) } \\
\text { Pulmonary fibrosis and emphysema (autopsy) }\end{array}$ & Unknown & $\begin{array}{l}\text { Unclassifiable fibrosis } \\
\text { (age } 42 \text { years) }\end{array}$ \\
\hline Lung cancer & "Lung cancer" & $\begin{array}{l}\text { Large cell carcinoma with myocardial } \\
\text { metastasis (autopsy) }\end{array}$ & "Lung cancer" & $\begin{array}{l}\text { Adenocarcinoma } \\
\text { (age } 45 \text { years) }\end{array}$ \\
\hline Outcome & Unknown & Death at age 23 years & Death at age 62 years & Death at age 46 years \\
\hline Familial disease & Yes & No & Yes & No \\
\hline Mutation & c.257dupA & c.859_860insC (de novo) & $\begin{array}{l}\text { c. } 650 \mathrm{C}>\mathrm{A} \text { (no DNA } \\
\text { available for the patient) }\end{array}$ & c.267dupg (de novo) \\
\hline
\end{tabular}

f)



b)

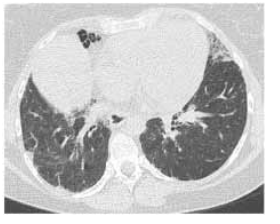

c)

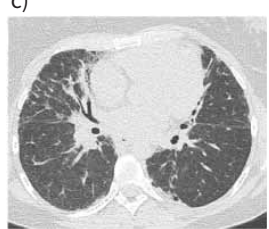

g)

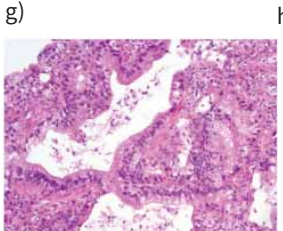

d)

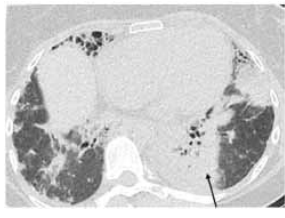

e)

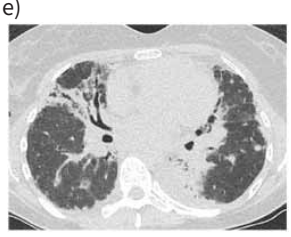

h)

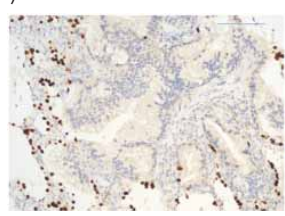

FIGURE 1 a) Reported cases of lung cancer associated with germline NKX2.1 mutation. b and c) Chest computed tomography (CT) image of a 42-year-old woman at interstitial lung disease (ILD) diagnosis, showing honeycombing associated with ground-glass opacities, reticulations and traction bronchiectasis with ventral and basal predominance. $d$ and e) Chest CT image at lung cancer diagnosis: emergence of several bilateral subpleural condensations. Arrow: transthoracic core biopsy. f) Evolution of pulmonary function test results after ILD diagnosis. Blue: forced vital capacity; red: forced expiratory volume in $1 \mathrm{~s}$; green: diffusing capacity of the lung for carbon monoxide; orange: carbon monoxide transfer coefficient. g) Transparietal biopsy: invasive mucinous adenocarcinoma (haematoxylin and eosin stain, original magnification 20x). h) Immunohistochemistry for thyroid transcription factor 1: positive for pneumocytes and negative for tumour cells (original magnification 20x). Scale bar= $100 \mu \mathrm{m}$.

$20 \mathrm{mmHg}$, cardiac output $8.5 \mathrm{~L} \cdot \mathrm{min}^{-1} \cdot \mathrm{m}^{-2}$ and pulmonary vascular resistance 4.7 Wood units. After multidisciplinary discussion, the diagnosis was unclassifiable pulmonary fibrosis [10].

The patient received azithromycin (250 mg three times a week) from July 2014 to December 2014 and prednisone $40 \mathrm{mg} \cdot$ day $^{-1}$ progressively tapered from December 2014 to June 2016. She did not show significant functional or radiological improvement. She also received first pirfenidone and then nintedanib in 2016 for a few weeks but experienced nausea and abdominal pain, without abnormal laboratory findings, and she decided to stop any antifibrotic therapy.

The disease was slowly progressive up to July 2019, when a chest CT scan showed several bilateral subpleural consolidations, with several nodules in the left lower lobe (figure $1 \mathrm{~b}-\mathrm{e}$ ). ${ }^{18} \mathrm{~F}$-fluorodeoxyglucose positron emission tomography-CT revealed five hypermetabolic nodules (maximal standardised uptake value 5.8) without any extrapulmonary involvement or mediastinal or hilar hypermetabolic lymphadenopathy. Cerebral magnetic resonance imaging findings were normal. Transthoracic core biopsy (arrow, figure 1d) showed invasive mucinous adenocarcinoma TTF1/ALK/ROS1/PD-L1 0\% (figure 1h). Next-generation sequencing did not reveal any driver somatic mutation/translocation associated with lung cancer (ALK, BRAF, EGFR, KRAS, c-MET exon 14, NRG1, NTRK1, NTRK3, RET and ROS1).

The patient underwent chemotherapy with carboplatin (area under the curve $5 \mathrm{mg} \cdot \mathrm{mL}^{-1} \cdot \mathrm{min}^{-1}$ ) and pemetrexed, followed by four cycles of maintenance pemetrexed. Lung cancer progressed locally with maintenance chemotherapy. In June 2020, she experienced an acute exacerbation, perhaps related to the chemotherapy, and received parenteral antibiotics and high-dose corticosteroids, but eventually died.

We describe a rare case of lung cancer and ILD associated with a germline NKX2.1 mutation. This case suggests a specific risk of lung cancer in adults with surfactant-associated gene mutations.

Lung cancer may develop in up to one third of patients with SFTPA1 and SFTPA2 germline mutations, which suggests a specific risk of lung cancer with these mutations $[3,11]$. ILD and lung cancer have been observed in the same family with SFTPA1 or SFTPA2 mutations but not necessarily in the same patient, so 
lung cancer development may be independent of the ILD [3, 11]. Ageing might be an important factor in the development of lung cancer in patients with surfactant-associated gene mutations. Indeed, SFTPA1 and SFTPA2 germline mutations are usually detected in adults (mean age 43 years), whereas many SFTPB, SFTPC, ABCA3 and NKX2.1 mutations have been mostly reported in children, and may not allow the necessary development time for cancer [12]. For instance, homozygous SFPTB and ABCA3 null mutations are associated with neonatal distress leading to death or lung transplantation before age 1 year [12]. Inhaled toxins, such as tobacco smoke, may be cofactors for carcinogenesis, as evidenced in our patient with a 10-year exposure to tobacco smoke.

Almost 150 patients with NKX2.1 mutation have been reported, 60\% with ILD [9]. The pathophysiology of ILD in patients with an NKX2.1 mutation is unknown but the main hypothesis relates to endoplasmic reticulum stress and caspase pathway activation in type II cells [13]. Corticosteroids, azithromycin and/or hydroxychloroquine might ameliorate the ILD related to SFTPC or ABCA3 mutation, but evidence in adults is lacking [14]. Because NKX2.1 interferes with SFTPC promoters, the same treatment may be effective [4]. Our patient received azithromycin and prednisone without objective improvement, as well as pirfenidone and nintedanib as fibrosis progressed but did not tolerate them. Prospective data and clinical trials are urgently needed to define better the optimal treatment for these patients. The patient had only a limited germline genetic analysis in 2014, which did not include RTEL1 and other telomere-related gene sequencing since associated with familial pulmonary fibrosis. Whether we should offer or repeat next-generation sequencing or whole-exome sequencing to families with suspected genetic cause of pulmonary fibrosis is an important question and is the subject of an ongoing dedicated European Respiratory Society task force.

No known addictive somatic mutation was evidenced in the cancer, which is another argument for an original carcinogenesis. Most frequent germline mutations associated with increased risk of lung cancer involve EGFR and p53 in Li-Fraumeni familial syndrome. In addition to SFTPA genes, NKX2.1 may be another germline risk factor for lung cancer, as supported by its role in the differentiation of the terminal respiratory unit cells and peripheral lung development, as a lineage-survival oncogene in lung adenocarcinoma and its recently evidenced crosstalk with EGFR/ERBB3 [15]. Indeed, lung cancer was reported in three other patients with NKX2.1 mutation, with almost no data about the lung cancer history in two cases (figure 1a) [6]. The third case was a 23-year-old man confirmed to carry an NKX2.1 mutation, with localised lung cancer associated with ILD, although the three diagnoses were established post mortem [7].

From a therapeutic point of view, the association of cancer and ILD is a major concern. Pre-existing ILD limits the possibility of surgery or radiotherapy [1]. Moreover, this unique pathophysiology does not suggest an effective targeted therapy or immunotherapy, as evidenced by negative next-generation sequencing findings and lack of PD-L1 expression.

Although we cannot rule out a coincidence, NKX2.1 mutation, as well as mutations in other surfactant-associated genes, is associated with ILD and possibly increased risk of lung cancer. A lung cancer screening strategy should be evaluated for these patients, with the balance of the risk of radiation exposure with repeated CT scanning and the difficulty of therapeutics in patients with ILD. Furthermore, owing to the rarity of lung transplantation for cancer, the conclusions that can be drawn about lung transplantation for this indication are limited. In addition, the ethical balance of how to allocate a scarce resource, such as a donor lung, remains an unresolved dilemma given the uncertainties regarding long-term survival [16].

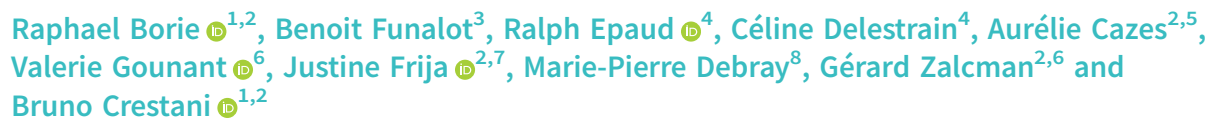

${ }^{1}$ Service de Pneumologie A, Hôpital Bichat, APHP, INSERM U1152 and Université de Paris, Paris, France. ${ }^{2}$ Laboratoire de Génétique, Hopital Henri Mondor, APHP, Creteil, France. ${ }^{3}$ Université Paris Est Creteil, INSERM, IMRB, Creteil, France. ${ }^{4}$ Service de Pédiatrie, Centre Hospitalier Intercommunal de Créteil, Créteil, France. ${ }^{5}$ Laboratoire d'Anatomopathologie, Hôpital Bichat, APHP, INSERM U1152 and Université de Paris, Paris, France. ${ }^{6}$ Service d'Oncologie thoracique and CIC1425 INSERM, Hôpital Bichat, APHP, INSERM U1152 and Université de Paris, Paris, France. ${ }^{7}$ Laboratoire de physiologie, Hôpital Bichat, APHP, INSERM U1152 and Université de Paris, Paris, France. ${ }^{8}$ Service de Radiologie, Hôpital Bichat, APHP, INSERM U1152 and Université de Paris, Paris, France. 
Provenance: Submitted article, peer reviewed.

Conflict of interest: R. Borie reports grants and personal fees from Boehringer Ingelheim and Roche, personal fees from Sanofi, and participation on a data safety monitoring/advisory board for Savara. B. Funalot has nothing to disclose. R. Epaud has nothing to disclose. C. Delestrain has nothing to disclose. A. Cazes has nothing to disclose. V. Gounant reports support for attending meetings and/or travel from AstraZeneca, BMS, Takeda and Pfizer, and participation on a data safety monitoring/advisory board for MSD, Chugai, Novartis, Boehringer and Takeda. J. Frija reports personal fees from Withings and Hinlab, and support for attending meetings and/or travel from Boehringer Ingelheim, Oxyvie, ADEP Assistance and Vitalaire, outside the submitted work. M.P. Debray reports personal fees from Boehringer Ingelheim and Roche outside the submitted work. G. Zalcman reports a research grant for a PhD thesis in his laboratory from Fondation Roche; consulting fees from BMS, AstraZeneca and Da Volterra; and support for attending meetings and/or travel from BMS, Abbvie, AstraZeneca and Pfizer. B. Crestani has nothing to disclose.

\section{References}

1 Tzouvelekis A, Spagnolo P, Bonella F, et al. Patients with IPF and lung cancer: diagnosis and management. Lancet Respir Med 2018; 6: 86-88.

2 Borie R, Kannengiesser C, de Fontbrune F S, et al. Management of suspected monogenic lung fibrosis in a specialised centre. Eur Respir Rev 2017; 26: 28446600.

3 Nathan N, Giraud V, Picard C, et al. Germline SFTPA1 mutation in familial idiopathic interstitial pneumonia and lung cancer. Hum Mol Genet 2016; 25: 1457-1467.

4 Guillot L, Carre A, Szinnai G, et al. NKX2-1 mutations leading to surfactant protein promoter dysregulation cause interstitial lung disease in "Brain-Lung-Thyroid Syndrome". Hum Mutat 2010; 31: E1146-E1162.

5 Hamvas A, Deterding RR, Wert SE, et al. Heterogeneous pulmonary phenotypes associated with mutations in the thyroid transcription factor gene NKX2-1. Chest 2013; 144: 794-804.

6 Gras D, Jonard L, Roze E, et al. Benign hereditary chorea: phenotype, prognosis, therapeutic outcome and long term follow-up in a large series with new mutations in the TITF1/NKX2-1 gene. J Neurol Neurosurg Psychiatry 2012; 83: 956-962.

7 Willemsen MA, Breedveld GJ, Wouda S, et al. Brain-Thyroid-Lung syndrome: a patient with a severe multi-system disorder due to a de novo mutation in the thyroid transcription factor 1 gene. Eur $\mathrm{J}$ Pediatr 2005; 164: 28-30.

8 Glik A, Vuillaume I, Devos D, et al. Psychosis, short stature in benign hereditary chorea: a novel thyroid transcription factor-1 mutation. Mov Disord Off J Mov Disord Soc 2008; 23: 1744-1747.

9 Nattes E, Lejeune S, Carsin A, et al. Heterogeneity of lung disease associated with NK2 homeobox 1 mutations. Respir Med 2017; 129: 16-23.

10 Borie R, Kannengiesser C, Gouya L, et al. Pilot experience of multidisciplinary team discussion dedicated to inherited pulmonary fibrosis. Orphanet J Rare Dis 2019; 14: 280.

11 van Moorsel $\mathrm{CH}$, Ten Klooster L, van Oosterhout MF, et al. SFTPA2 mutations in familial and sporadic idiopathic interstitial pneumonia. Am J Respir Crit Care Med 2015; 192: 1249-1252.

12 Bullard JE, Wert SE, Whitsett JA, et al. ABCA3 mutations associated with pediatric interstitial lung disease. Am J Respir Crit Care Med 2005; 172: 1026-1031.

13 Mulugeta S, Maguire JA, Newitt JL, et al. Misfolded BRICHOS SP-C mutant proteins induce apoptosis via caspase-4- and cytochrome c-related mechanisms. Am J Physiol Lung Cell Mol Physiol 2007; 293: L720-L729.

14 Kroner C, Wittmann T, Reu S, et al. Lung disease caused by ABCA3 mutations. Thorax 2017; 72: 213-220.

15 Yamamoto $\mathrm{H}$, Yatabe $\mathrm{Y}$, Toyooka S. Inherited lung cancer syndromes targeting never smokers. Transl Lung Cancer Res 2018; 7: 498-504.

16 Glanville AR, Wilson BE. Lung transplantation for non-small cell lung cancer and multifocal bronchioalveolar cell carcinoma. Lancet Oncol 2018; 19: e351-e358. 